\title{
Pseudomonas otitidis
}

National Cancer Institute

\section{Source}

National Cancer Institute. Pseudomonas otitidis. NCI Thesaurus. Code C128547.

A species of Gram-negative, motile, bacillus-shaped bacteria in the phylum

Proteobacteria. The species was isolated from patients with otic infections. 